\title{
Lag Screw Fixation for Treatment of an Ulnar Styloid Process Fracture with Antebrachiocarpal Joint Luxation in a Cat
}

\author{
Lindsay A. Parker ${ }^{1}$ Amy S. Kapatkin ${ }^{2}$ Mark C. Fuller ${ }^{3}$ \\ ${ }^{1}$ Department of Companion Animals, Atlantic Veterinary College, \\ University of Prince Edward Island, Charlottetown, Canada \\ 2 Department of Surgical and Radiological Sciences, University of \\ California, Davis, CA, United States \\ 3 Fuller Animal Specialty Surgery, Airdrie, AB, Canada \\ Address for correspondence Lindsay A. Parker, DVM, Atlantic \\ Veterinary College, University of Prince Edward Island, 550 University \\ Avenue, Charlottetown, PEI, C1A 4P3, Canada \\ (e-mail: lap177@usask.ca).
}

VCOT Open 2019;2:e32-e35.

\author{
Abstract \\ Keywords \\ - feline ulnar styloid \\ fracture \\ - osteosynthesis
}

Fracture of the ulnar styloid process can result in clinically debilitating antebrachiocarpal instability. Previously described repair methods include Kirschner wire and figure of eight tension band wire and/or intramedullary pinning. This case report describes surgical repair of a short oblique ulnar styloid process fracture in a cat using two cortical screws placed in lag fashion. Recheck evaluation confirmed lameness-free full return to function with anatomical fracture healing.

\section{Introduction}

Lateral styloid process fractures generally require surgical stabilization to avoid carpal instability and to act as a physical buttress to provide normal joint function. ${ }^{1}$ Due to the small diameter of the feline distal ulna, these types of fractures have traditionally been repaired with one Kirschner wire coupled with a figure of eight tension band. ${ }^{2,3}$ The purpose of this mode of internal fixation is to achieve adequate fragment compression and fixation. The technique is more easily employed in the canine patient, whereas in the cat, given the small size of bone fragments, this can be challenging. We describe in this case report an alternative method using a lag screw technique that resulted in anatomical fracture alignment and healing without complication.

\section{Case Description}

A 3-year-old, male neutered, $5.4 \mathrm{~kg}$, domestic short-haired cat was presented for an acute right thoracic limb lameness after having been attacked by the owner's dog. The patient exhibited a non-weight bearing lameness on the right thor-

(1DLindsay A. Parker's ORCID is https://orcid.org/0000-0001-83079807.

received

December 22, 2018

accepted after revision

April 7, 2019 acic limb and, on examination under sedation, was found to have a marked soft tissue swelling associated with the distal right antebrachium and increased lateral carpal laxity.

Radiographs revealed an antebrachiocarpal joint luxation with an oblique ulnar styloid process fracture (-Fig. 1). The superficial bite wound on the right carpus was cleaned and closed primarily. A spoon splint was placed to stabilize the dislocated carpus until the patient could be evaluated for surgical repair. The following medications were prescribed $0.02 \mathrm{mg} / \mathrm{kg}$ buprenorphine (Vetergesic; Champion Alstoe, Richmond, Virginia, United States) PO q8h, and clindamycin $6 \mathrm{mg} / \mathrm{kg}$ (Clinacin; Merck Animal Health, Kirkland, Quebec, Canada) PO q12h, and Fortiflora probiotic powder (Purina, St. Louis, Missouri, United States) PO q24h for diarrhoea that developed while the cat was hospitalized, suspected to be secondary to stress and antibiotic administration.

The patient was evaluated 5 days later. The diarrhoea had resolved, food intake had returned to normal, but the cat had removed the splint. The patient was found to be partial weight bearing on the right thoracic limb with marked valgus deviation of the carpus. The remainder of the examination was performed under sedation. On palpation, the carpus was unstable with crepitus and laxity laterally. The medial
License terms Stuttgart · New York
DOI https://doi.org/

10.1055/s-0039-1691827. ISSN 2625-2325.

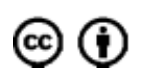




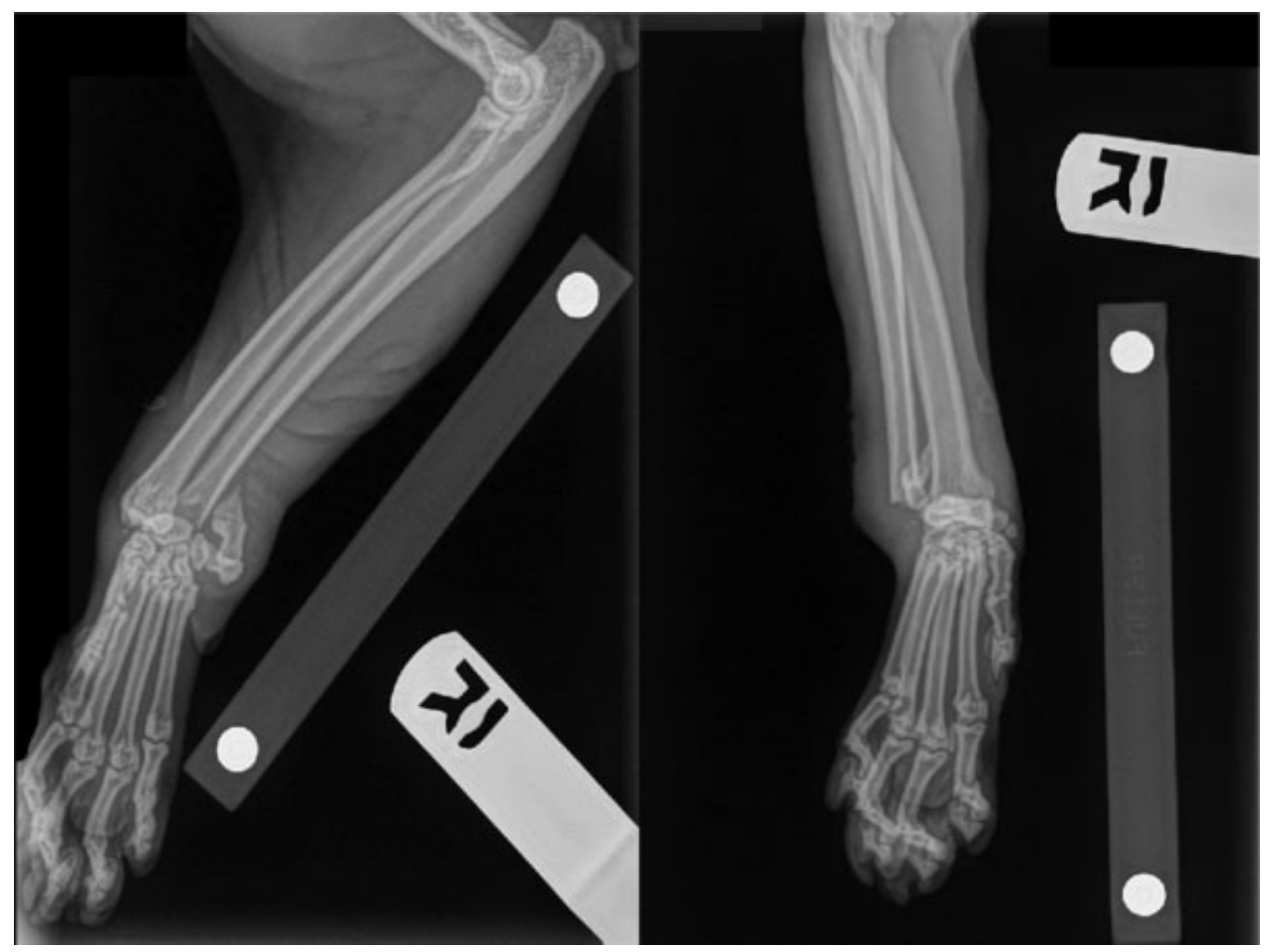

Fig. 1 Initial patient radiographs confirming an oblique ulnar styloid fracture with antebrachiocarpal joint luxation.

collateral ligament appeared to be intact. No other orthopaedic abnormalities were identified and the superficial wounds appeared to be healing well. Open reduction and internal fixation were planned. Pre-operative bloodwork revealed mild hyperglycaemia (13.64 $\mathrm{mmol} / \mathrm{L}, 4.11-8.84$ ) and mild non-regenerative anaemia (29.7\%, 30.3-52.3), which was deemed to be clinically insignificant. The patient was placed under general anaesthesia. The fracture was approached laterally over the styloid ulnar process and was reduced and stabilized with two $1.5-\mathrm{mm}$ cortical screws (DePuy Synthes; Johnson \& Johnson, Guelph, Ontario, Canada) placed perpendicularly to the fracture line in lag fashion to achieve inter-fragmentary compression.

Postoperative radiographs confirmed anatomic fracture fragment reduction and correct implant placement ( - Fig. 2). The cat was hospitalized with intravenous fluids and 0.05

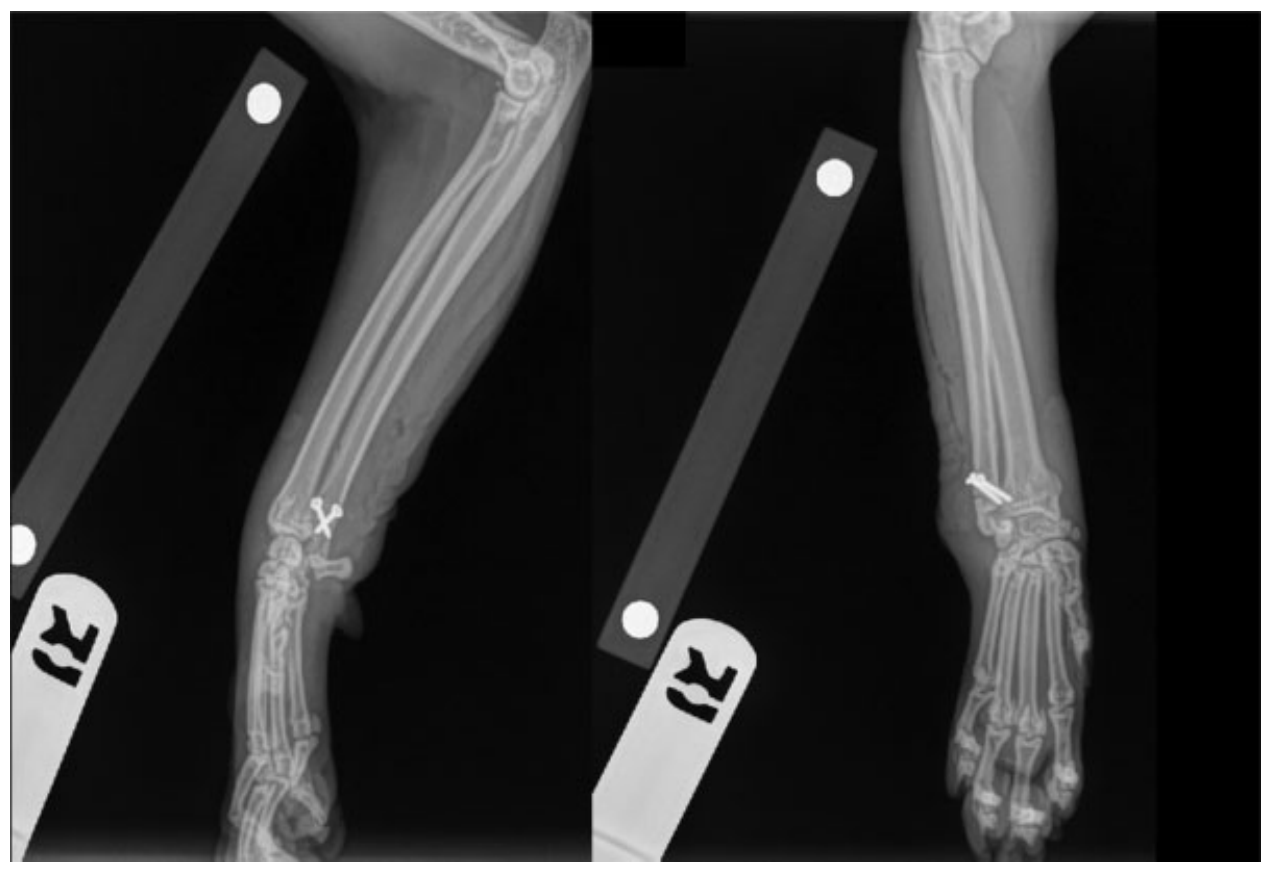

Fig. 2 Immediate postoperative radiographs. 


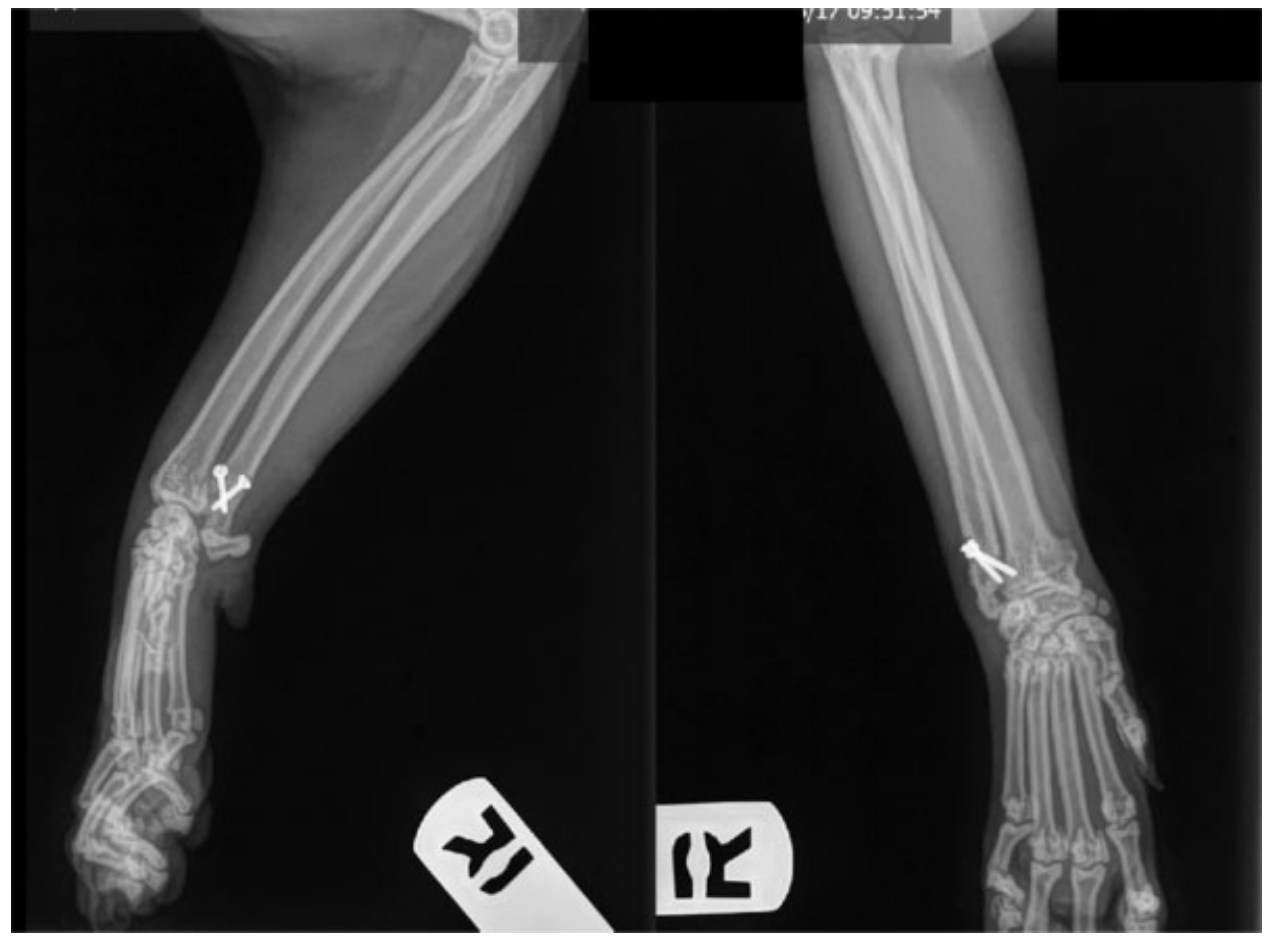

Fig. 3 Follow-up radiographs 2 years following fracture reduction.

$\mathrm{mg} / \mathrm{kg}$ hydromorphone (Pfizer, Kirkland, Quebec, Canada) intravenously administered $\mathrm{q} 4-6 \mathrm{~h}$ and was discharged the following day with $0.02 \mathrm{mg} / \mathrm{kg}$ buprenorphine $\mathrm{PO} \mathrm{q} 8 \mathrm{~h}$ (Vetergesic; Champion Alstoe, Richmond, Virginia, United States) and the previously prescribed clindamycin was discontinued as an $8 \mathrm{mg} / \mathrm{kg}$ dose of cefovecin (Convenia; Zoetis, Kalamazoo, Michigan, United States) was administered subcutaneously (due to patient demeanour and the reported challenges administering oral medications). Confinement for 8 weeks was prescribed. Although postoperative radiographs were scheduled, the client did not return for re-examination until 2 years later. At that time, the cat was doing well clinically with no lameness, pain or atrophy of the limb. Radiographs taken at that time revealed clinical union of the previously noted fracture site with stable implants and only mild carpal osteoarthritis at the level of the antebrachiocarpal joint (-Fig. 3).

\section{Discussion}

This is the first report documenting surgical repair of an ulnar styloid fracture with two lag screws in a feline patient. Antebrachial fractures of the forelimb typically involve a fractured radius with concurrent ulnar fracture. Due to transmission of load forces between the carpus and the elbow via the radius, these fractures are commonly stabilized by means of internal fixation of the radius only. ${ }^{2}$ In unusual situations, isolated fractures of the ulnar styloid process can occur with carpal trauma, independent of the radius. ${ }^{2}$

The lateral styloid process of the ulna serves as the proximal attachment of the short lateral collateral ligament of the antebrachiocarpal joint and extends distal to the proximal row of carpal bones, thereby providing a buttress for lateral carpal stability. ${ }^{2,3}$ Therefore, when an ulnar styloid fracture arises it must be addressed to avoid carpal subluxation, carpal valgus and lameness associated with fracture mal-union, nonunion or osteoarthritis. ${ }^{3}$ Ulnar styloid fractures may present as partial or complete fractures and previously described fixation techniques have included a laterally placed plate or placement of a single intramedullary pin or Kirschner wire coupled with a figure of eight tension band. Reportedly, partial styloid fractures may also be managed conservatively with a splint and bandage for 2 to 3 weeks. ${ }^{4}$ However, non-rigid bone union heals by secondary intention, entailing callus formation; thus, development of degenerative carpal joint disease may thereby be enhanced.

Therefore, direct bone healing seems advantageous in this described carpal-near form of fracture. ${ }^{3}$ During direct contact healing, bone union and bone remodelling occur simultaneously. ${ }^{3}$ With the combination of precise reduction and rigid fixation, as with compression plates or lag screws, osteoprogenitor cells are not signalled to mobilize to the fracture site and thus, a callous does not occur as seen with secondary gap healing. ${ }^{3}$ We therefore felt that osteosynthesis by rigid compression is advantageous to treat ulnar styloid fractures to minimize callous formation and to decrease the risk of later osteoarthrosis development.

The lag screw technique can be applied with long or short oblique fractures; by placing screws in a lag fashion it adequately opposes the oblique fracture shearing force and therefore aids in the overall strength of the construct. ${ }^{3}$ To achieve maximum interfragmentary compression, the lag screw is placed in the middle of the fragment directed at a right angle to the fracture plane to provide a stable interdigitation of fracture fragments. ${ }^{3}$ If not placed perpendicular to the fracture line, the fragments will become displaced as the lag screw is 
tightened. ${ }^{3}$ In the described case, two lag screws were placed in divergent fashion to act perpendicularly to the fracture line and to increase the stability of the repair by opposing counterrotational forces. This decision was made intra-operatively as the patient's small bone size was a limiting factor for implant placement. Another internal fixation option could have been one lag screw with a tension wire, which has not been previously described in the cat. In dogs, ulnar styloid fractures are typically reduced using one or more Kirschner pins and tension band combination. ${ }^{2,3}$ However, if only one Kirschner pin can be placed due to the small size of the fragment, then external coaptation support is recommended postoperatively due to risk of rotational instability. ${ }^{1}$ While we also could have applied a tension band technique, our patient was known not tolerating any external coaptation and therefore was deemed an unsuitable candidate for the tension band technique. Additionally, in a cat there is limited soft tissue coverage over the styloid process and as tension band wires cannot be countersunk, they can cause irritation of the soft tissues with the risk of penetrating the skin. Taking into account these considerations, a more rigid fracture reduction was chosen as alternative to the tension band technique. This also, because placement of lag screws helps to avoid post-operative coaptation and the relative risk of later implant removal, should implant migration and/or failure occur, which can be seen with Kirschner wire and tension band technique.

With the herein described technique, the patient did not experience any complications requiring surgical revision. From the initial postoperative radiograph to follow-up radiographs taken at 2 years, there was development of mild osteoarthritis at the level of the antebrachiocarpal joint. This may have been due to lack of postoperative rest as the cat was difficult to handle. This patient was already at an increased risk of osteoarthritis development, described as post-traumatic osteoarthritis; hence, it may be unrelated to our fixation technique. ${ }^{4}$ This assumption is supported by a study by Gordon and colleagues, which showed that in humeral condylar fractures, despite near perfect anatomical reduction of articular surfaces, osteoarthrosis had progressed on follow-up radiographs in all cases evaluated over a 43 -month period. ${ }^{5}$ Possible complications associated with the lag screw technique includes inadequate screw placement resulting in an insufficient anatomical alignment leading to chronic joint instability and osteoarthritis development, as well as implant failure and/or infection.

This single case indicates that in the cat, oblique ulnar styloid fractures may be managed via placement of lag screws, allowing anatomical reduction with rigid stability, resulting in uncomplicated fracture healing without the need of external coaptation.

Author Contribution

This manuscript has been seen and approved by all authors.

Conflict of Interest

None declared.

\section{References}

1 Johnson A, Dunning D. Atlas of Orthopedic Surgical Procedures of the Dog and Cat. 1st ed. St. Louis, Missouri: Elsevier; 2005:30

2 Johnston SA, Tobias KM, eds. Veterinary Surgery Small Animal. 2nd ed. St. Louis, Missouri: Elsevier; 2018920

3 Johnson AL, Houlton JEF, Vannini R, eds. AO Principles of Fracture Management in the Dog and Cat. 1st ed. AO Publishing, Switzerland: Thieme; 2005:31, 255, 268

4 Montavon PM, Voss K, Langley-Hobbs SJ. Feline Orthopedic Surgery and Musculoskeletal Disease. 1st ed. Philadelphia, Pennsylvania: Saunders Elsevier; 2009:381-382

5 Gordon WJ, Besancon MF, Conzemius MG, Miles KG, Kapatkin AS, Culp WTN. Frequency of post-traumatic osteoarthritis in dogs after repair of a humeral condylar fracture. Vet Comp Orthop Traumatol 2003;16:1-5 